\title{
Post-warming survival rates and clinical outcomes of human cleavage stage embryos vitrified/warmed using CryoTouch and Cryotop methods
}

\author{
Somayeh Keshavarzi ${ }^{1}$, Azadeh Dokht Eftekhari², Hajar Vahabzadeh², Marzieh Mehrafza², \\ Robabeh Taheripanah",3, Masoumeh Asgharnia ${ }^{4}$, Sahar Esfandyari, ${ }^{1,5}$, Alaleh Ghazifard', \\ Hossein Hosseinirad ${ }^{1 *}$ (1) and Shahrokh Paktinat ${ }^{1 *}$ (I)
}

\begin{abstract}
Background: Vitrification has become the method of choice for cryopreservation of human embryos and gametes. There are multiple commercial media, containing different combinations and concentrations of cryoprotectants, available for vitrification and warming procedures. The aim of this retrospective study was to compare post-warming survival rate and clinical outcomes of cleavage stage embryos vitrified/warmed using two different commercial methods (CryoTouch and Cryotop) during intracytoplasmic sperm injection/frozen embryo transfer (ICSI/FET) cycles. This retrospective study evaluated a total of 173 FET cycles performed on 446 warmed cleavage stage embryos between January 2018 and December 2020. Post-warming embryo survival rate and clinical outcomes including clinical pregnancy, implantation, and live birth rates were calculated.

Results: The results showed no significant differences between two groups in terms of post-warming survival rate ( $p$ value $=0.5020)$, clinical pregnancy rate $(p$ value $=0.7411)$, implantation rate $(p$ value $=0.4694)$, and live birth rate $(p$ value $=0.5737$ )

Conclusions: Collectively, high successful rates were observed in outcomes of vitrified/warmed cleavage stage embryos using both CryoTouch and Cryotop commercial methods.
\end{abstract}

Keywords: Cryopreservation, Embryo vitrification, Survival rate, Clinical outcome

\section{Background}

Production of excess embryos is prevailing during in vitro fertilization/intracytoplasmic sperm injection (IVF/ICSI) cycles. The embryos that are not selected for transfer to the uterus should be cryopreserved to be used in future embryo transfer (ET) cycles [1]. Therefore, embryo cryopreservation provides additional chances for achieving

\footnotetext{
*Correspondence: rad6438@gmail.com; sh.paktinat@gmail.com ${ }^{1}$ Infertility Clinic, Erfan Niayesh Hospital, Iran University of Medical Sciences, Tehran, Iran

Full list of author information is available at the end of the article
}

pregnancy without extra exposures to the exogenous gonadotropins used for ovarian stimulation [2]. In addition, embryo cryopreservation facilitates the single embryo transfer (SET) program performed to avoid multiple pregnancies $[2,3]$. Frozen embryo transfer (FET) cycles are now preferred over fresh ET cycles as they avoid to transfer the embryo into the uterus if the endometrial preparation is suboptimal [4]. The first report of successful pregnancy achieved by a FET cycle was published in 1983 [5]. Since then, embryo cryopreservation has become an undeniable part of the assisted reproductive technology (ART) $[4,6]$. 
A successful cryopreservation procedure preserves all the functional and morphological properties of embryonic cells and protects them against damages caused by ice crystal formation and fracture damages, the main concerns during steps of cryopreservation, i.e., cooling and warming [7]. Different cryoprotective agents (CPAs) and cooling-warming rates are being used to avoid detrimental events [8]. There are two common methods for embryo and gamete cryopreservation in ART practice, slow freezing and vitrification [7]. Over the past decades, vitrification has gradually become the method of choice for embryo and gamete cryopreservation because of ease of use and low cost $[9,10]$. Vitrification has been broadly used for human cleavage and blastocyst stage embryos $[6,11]$.

Vitrification eliminates ice crystal growth by ultrarapid freezing of the cells in a mixture of concentrated CPAs [7]. It has been well established that embryo survival and clinical outcomes are improved by vitrification $[7,12]$. In addition, vitrification needs no expensive equipment and requires less time in comparison to the slow freezing [7, 12]. Based on these reasons, vitrification is being used by almost every fertility treatment center worldwide [13, 14].

A great variation exists in vitrification protocols, most of which are cryopreservation devices $\left(\right.$ Cryotop ${ }^{\circledR}$, CryoTip $^{\circledR}$, Cryolock $^{\circledR}$, Cryoloop, Open Pulled Straw, High Security Vitrification Straw, etc.); however, the acceptable result largely depends on the quality of the embryos, the duration of exposure to CPAs, and reduced volume of the solution used for loading the embryos on the device [15]. As the vitrification device and media (solutions) all have enormous impacts on the final results, some studies compare different vitrification methods [16-18]. Herein, the vitrification method means the use of a vitrification device and vitrification/warming media from the same manufacturer, and this nomenclature is often based on the name of the vitrification device.

Most of the previous studies have focused on identifying the best cryopreservation method with the highest clinical yields $[7,12,14]$. Although there are multiple commercial vitrification/warming methods available for ART use, no effort has been made to compare the benefits/risks and cost/efficiency ratios among them so far $[15,19]$. It is assumed that products provided by different companies may differ in terms of advantages and limitations. In addition, the availability of the product for ART clinics may also impact the clinical results due to the instability of their composition, especially during transit.

The aim of this retrospective study was to evaluate the post-warming survival rate and clinical outcomes of cleavage stage embryos vitrified/warmed using two commercial methods: CryoTouch method (RS Medical, Ravan
Sazeh Co., Tehran, Iran) and Cryotop method (Kitazato BioPharma Co., Shizuoka, Japan).

\section{Methods \\ Study population}

This retrospective study was conducted to analyze the survival rate of cleavage stage embryos after vitrification/warming performed by CryoTouch and Cryotop methods, and the subsequent clinical outcomes in two infertility clinics located at Erfan Niayesh Hospital Tehran, Iran and Mehr Fertility Research Center, Rasht, Iran, from January 2018 to December 2020. The study protocol was done according to the Ethical Principles for Medical Research and approved by the Institutional Review Board of each center.

The details about patients' basic characteristics, protocol of ovarian stimulation, ICSI procedure, embryo culture and evaluation methods, vitrification and warming protocols, FET protocol, and clinical outcomes were all obtained from registered documents at the mentioned infertility clinics. A total of 978 FET cycles involving embryos vitrified/warmed on day 3 of development were evaluated to enter the study based on the following inclusion/exclusion criteria:

The inclusion criteria were age range between 20 and 35 years old, body mass index (BMI) $<30 \mathrm{~kg} / \mathrm{m}^{2}$, serum level of follicle-stimulating hormone (FSH) $<10 \mathrm{mIU} / \mathrm{ml}$ on day 3 of menstrual cycle, having more than 8 cleavage stage embryos produced by ICSI procedure, and undergoing the first ICSI/FET cycle with gonadotropin-releasing hormone $(\mathrm{GnRH})$ antagonist protocol for ovarian stimulation.

The exclusion criteria included the presence of anatomical uterine anomalies, space-occupying lesions, hormonal dysregulations, ovarian hyperstimulation syndrome (OHSS), inflammatory disorders, hydrosalpinx, endometriosis, autoantibodies, history of ectopic pregnancy, repeated implantation failure (RIF), miscarriage, and having less than 8 cleavage stage embryos produced by ICSI procedure.

\section{Ovarian stimulation protocol}

GnRH antagonist protocol was performed in all patients. Table 2 provides ovarian stimulation characteristics and final outcomes in patients undergoing an ovarian stimulation procedure. First, estradiol valerate (2 mg, PO, BID; Aburaihan Co., Tehran, Iran) was administered from day 21 of the natural cycle and continued until days 2-4 of the subsequent cycle. Then, follicular growth was stimulated by administration of recombinant FSH (150-225 IU, daily; Gonal F, Merck, Germany) from day 2 or 3 of the cycle. The prescribed dose was adjusted based on the follicular growth 
monitored using transvaginal ultrasonography. GnRH antagonist (Cetrotide, Merck, Germany) was administrated when the dominant follicles reached the size of $13-14 \mathrm{~mm}$ in diameter. GnRH antagonist administration was continued until the day of ovulation induction. Human chorionic gonadotropin (hCG, 10,000 IU; Choriomon, IBSA, Switzerland) was injected to induce oocyte maturation. Transvaginal ultrasound-guided ovum pick-up (OPU) was performed $36 \mathrm{~h}$ after hCG injection.

\section{Cumulus cell-oocyte complex retrieval, oocyte denudation, and fertilization}

All the used culture media were supplemented with $10 \%$ $(\mathrm{V} / \mathrm{V})$ of human serum albumin (HSA) and were equilibrated for $8 \mathrm{~h}$ at $37{ }^{\circ} \mathrm{C}$ in $6 \% \mathrm{CO}_{2}$ incubator before use. The retrieved cumulus cell-oocyte complexes (COCs) were washed in a handling medium (HTF w/HEPES, Fertilite $^{\circledR}$, Ravan Sazeh Co., Tehran, Iran ) and maintained in embryo culture medium (SingleCulture Medium, Fertilite ${ }^{\circledR}$, Ravan Sazeh Co., Tehran, Iran ) for $2 \mathrm{~h}$ at $37^{\circ} \mathrm{C}$ in $6 \% \mathrm{CO}_{2}$ incubator prior to denudation. Oocyte denudation was conducted using both enzymatic (Hyaluronidase, Fertilite ${ }^{\circledR}$, Ravan Sazeh Co., Tehran, Iran) and mechanical methods. ICSI procedure was performed on all matured oocytes (metaphase II stage) 3-4 h after OPU. Then, the injected oocytes were cultured in 30-50 $\mu \mathrm{L}$ of embryo culture medium (SingleCulture Medium, Fertilite $^{\circledR}$, Ravan Sazeh Co., Tehran, Iran) under mineral oil (RS Medical, Ravan Sazeh Co., Tehran, Iran) overlay. On day 3 of development, the resulting embryos were assessed morphologically and high-quality embryos were selected for vitrification.

\section{Cleavage stage embryo quality evaluation}

Cleavage stage embryo quality was assessed by recording morphological parameters according to the literature [20]. The morphological parameters included the number and symmetry of blastomeres, degree of fragmentation (anucleate structures of blastomeric origin), presence of multinucleation, and presence of abnormalities in intracytoplasmic and extracytoplasmic compartments. Highquality cleavage stage embryos were defined as those with 4 blastomeres on day 2 or 6-8 blastomeres on day 3 , having symmetric blastomeres, having less than $15 \%$ fragmentation, without multinucleation, without intracytoplasmic abnormalities such as vacuoles and inclusion bodies, and without extracytoplasmic abnormalities such as granularities in the perivitelline space and zona pellucida dysmorphism. Otherwise, the embryos were considered as low-quality embryos.

\section{Vitrification and warming procedures}

High-quality cleavage stage embryos were selected for the vitrification with two commercial methods and the procedure was performed based on the manufacturer's protocol, CryoTouch method (RS Medical, Ravan Sazeh Co., Tehran, Iran) or Cryotop method (Kitazato BioPharma Co., Shizuoka, Japan). The method means the use of vitrification/warming media and vitrification devices from the same manufacturer. CryoTouch and Cryotop methods had similar protocols for vitrification and warming procedures with a little difference. Briefly, the equilibration was performed in equilibration solution for $7 \mathrm{~min}$ in CryoTouch method or $10 \mathrm{~min}$ in Cryotop method, both at room temperature. Afterwards, the embryos were placed in vitrification solution for 50-60 $\mathrm{s}$ in both protocols. Then, the embryos (maximum 3 in each vitrification device) were immediately aspirated with a minimum volume of the vitrification solution and placed onto the tip of CryoTouch ${ }^{\circledR}$ or Cryotop ${ }^{\circledR}$ vitrification devices. The loaded devices were immediately submerged vertically into liquid nitrogen, then placed in a goblet and stored in a liquid nitrogen tank.

In the morning of FET, the embryos were warmed in the corresponding commercial media based on the manufacturer's protocol. Briefly, the vitrification device was quickly removed from liquid nitrogen and immersed in a warming solution (prewarmed at $37^{\circ} \mathrm{C}$ ). The embryos were detected and immediately transferred to another droplet of warming solution and incubated for $1 \mathrm{~min}$. Subsequently, the embryos were transferred to the dilution solution and incubated for $3 \mathrm{~min}$. In the next step, the embryos were transferred to the washing solution and maintained for $5 \mathrm{~min}$. Ultimately, the embryos were placed in embryo culture medium and incubated at $37^{\circ} \mathrm{C}$ in $6 \% \mathrm{CO}_{2}$ until ET.

\section{Embryo survival assay}

The vitrified/warmed embryos were considered survived if they had $50 \%$ or more viable blastomeres with no evidence of degenerated morphology.

\section{FET cycle}

Hormone replacement therapy (HRT) was performed for preparing the endometrium as a standard protocol. Estradiol valerate $(6 \mathrm{mg} /$ day, PO; Aburaihan Co., Tehran, Iran) was administered from days $2-3$ of the menstrual cycle and continued up to $8 \mathrm{mg} /$ day until the endometrial thickness reached 8 millimeters. Progesterone (400 mg, suppository, BID; Cyclogest, Actavis, England, UK) was initiated when the endometrial thickness was upper $8 \mathrm{~mm}$. In the presence of a positive result for $\beta$-hCG test, the estradiol and progesterone 
administrations were continued until weeks 6 and 12 of gestation, respectively.

ET was conducted using an embryo transfer catheter (Guardia $^{\mathrm{TM}}$ Access, Cook, USA) by an expert gynecologist under the guidance of ultrasound, based on the guideline provided by the American Society for Reproductive Medicine (ASRM). Single or double high-quality cleavage-stage vitrified/warmed embryos were selected for each ET cycle.

\section{Outcome assessment}

The embryo post-warming survival rate was calculated as the percentage of survived embryos, based on the mentioned definition, after the warming procedure.

The clinical pregnancy rate was calculated from the number of observed gestational sacs by ultrasonography per embryo transfer.

The implantation rate was calculated from the number of observed gestational sacs by ultrasonography per the number of transferred embryos.

The live birth rate was calculated from the number of live births per embryo transfer.

\section{Statistical analysis}

GraphPad Prism (GraphPad Software, USA) was used to analyze all the obtained data. Comparisons of the means were performed by Student's $t$ test. The $P$ value $<0.05$ was considered as the level of significance. Data are represented as mean \pm standard deviation (SD).

\section{Results}

A total of 173 ICSI/FET cycles have entered the study based on the inclusion/exclusion criteria. Among these cycles, 95 vitrification/warming cycles were conducted by the CryoTouch method and 78 vitrification/warming cycles were performed using the Cryotop method. As shown in Table 1, there were no significant differences between the two groups in mean age, BMI, duration of infertility, type of infertility (primary or secondary), serum level of day $3 \mathrm{FSH}$, serum level of anti-Mullerian hormone (AMH), and sperm count of the spouse.

Table 2 represents the characteristics of the studied patients during ovarian stimulation including the total dose of administered gonadotropin, duration of gonadotropin administration, serum levels of luteinizing

Table 1 Basic characteristics of the studied groups

\begin{tabular}{llll}
\hline Characteristics & Groups & & $p$ value \\
\cline { 2 - 3 } & Cryotop method & Cryotouch method & - \\
\hline Number of ICSI/FET cycles & 78 & 95 & NS \\
Age (years) & $31.11 \pm 3.75$ & $29.61 \pm 4.06$ & NS \\
BMl & $23.69 \pm 3.21$ & $24.87 \pm 3.65$ & NS \\
Duration of infertility (years) & $2.3 \pm 2.5$ & $2.6 \pm 1.9$ & NS \\
Type of infertility (primary) & $83.5 \%$ & $80.3 \%$ & NS \\
Type of infertility (secondary) & $16.5 \%$ & $19.7 \%$ & NS \\
FSH (day 3) (mlU/ml) & $5.9 \pm 3.8$ & $6.4 \pm 2.6$ & NS \\
AMH (ng/ml) & $4.25 \pm 2.23$ & $3.87 \pm 2.49$ & NS \\
Sperm (count/ml) & $(69 \pm 55) \times 10^{6}$ & $(50 \pm 45) \times 10^{6}$ & \\
\hline
\end{tabular}

$A M H$ anti-Mullerian hormone, $B M I$ body mass index, $F S H$ follicle-stimulating hormone, $N S$ non-significant

Table 2 Ovarian stimulation characteristics and outcomes in the studied groups

\begin{tabular}{|c|c|c|c|}
\hline \multirow[t]{2}{*}{ Characteristics } & \multicolumn{2}{|l|}{ Groups } & \multirow[t]{2}{*}{$p$ value } \\
\hline & Cryotop method & CryoTouch method & \\
\hline Total dose of administered gonadotropin (IU) & $1947.34 \pm 123.65$ & $1886.57 \pm 323.56$ & NS \\
\hline Duration of gonadotropin administration (days) & $9.3 \pm 2.1$ & $9.7 \pm 1.3$ & NS \\
\hline $\mathrm{LH}(\mathrm{IU} / \mathrm{mL})$ on day of trigger & $3.89 \pm 0.6$ & $4.17 \pm 0.5$ & NS \\
\hline E2 $(\mathrm{pg} / \mathrm{mL})$ on day of trigger & $2976.47 \pm 98.2$ & $3131.23 \pm 101.2$ & NS \\
\hline $\mathrm{P}(\mathrm{IU} / \mathrm{mL})$ on day of trigger & $0.96 \pm 0.3$ & $1.07 \pm 0.2$ & NS \\
\hline Number of retrieved COCs & $14.6 \pm 4.3$ & $11.2 \pm 5.2$ & NS \\
\hline High-quality cleavage stage embryos & $75 \%$ & $69 \%$ & NS \\
\hline
\end{tabular}

COCS cumulus cell-oocyte complexes, E2 estradiol, $L$ H luteinizing hormone, $N S$ non-significant, $P$ progesterone 
hormone (LH), estradiol (E2), and progesterone (P) on trigger day, number of retrieved COCs, and percent of high-quality cleavage stage embryos resulted from ICSI procedure. Based on the obtained data, there were no significant differences in these characteristics between the patients who were undergone ICSI/FET cycles using the CryoTouch or Cryotop methods.

A total of 446 cleavage stage embryos were warmed and 173 ET cycles were performed using double high-quality embryos. Figure 1 demonstrates the warmed embryos in two groups. Among 446 embryos, 233 embryos were vitrified/warmed using the CryoTouch method and 213 embryos were vitrified/warmed using the Cryotop method.

Post-warming survival rates of cleavage stage embryos were 89.57 \% for the CryoTouch method (95\% CI 85.3293.82) and $91.63 \%$ for the Cryotop method (95\% CI 87.35-95.91), with no significant difference between the groups $(p$ value $=0.5020)$ (Table 3$)$.

Clinical pregnancy rates were $43.16 \%$ for the CryoTouch method (95\% CI 29.33-56.98) and 39.74\% for the Cryotop method (95\% CI 24.61-54.87), with no
Table 3 Post-warming survival rate and clinical outcomes of vitrified/warmed cleavage stage embryos in the studied groups

\begin{tabular}{lllc}
\hline Variables & \multicolumn{2}{l}{ Groups } & p value \\
\cline { 2 - 3 } & $\begin{array}{l}\text { Cryotop } \\
\text { method } \\
(\boldsymbol{n}=\mathbf{7 8})\end{array}$ & $\begin{array}{l}\text { CryoTouch } \\
\text { method } \\
(\boldsymbol{n}=\mathbf{9 5})\end{array}$ & \\
\hline Number of warmed embryos & 213 & 233 & - \\
Post-warming survival rate & $91.63 \%$ & $89.57 \%$ & 0.5020 \\
Clinical pregnancy rate & $39.74 \%$ & $43.16 \%$ & 0.7411 \\
Implantation rate & $23.08 \%$ & $26.84 \%$ & 0.4694 \\
Live birth rate & $25.64 \%$ & $30.53 \%$ & 0.5737 \\
\hline
\end{tabular}

significant difference between the groups $(p$ value $=$ 0.7411) (Table 3).

Implantation rates were $26.84 \%$ for the CryoTouch method (95\% CI 19.90-33.78) and 23.08\% for the Cryotop method (95\% CI 15.43-30.72), with no significant difference between the groups ( $p$ value $=0.4694)($ Table 3$)$.

Live birth rates were $30.53 \%$ for CryoTouch method (95\% CI $18.61-42.44$ ) and $25.64 \%$ for the Cryotop
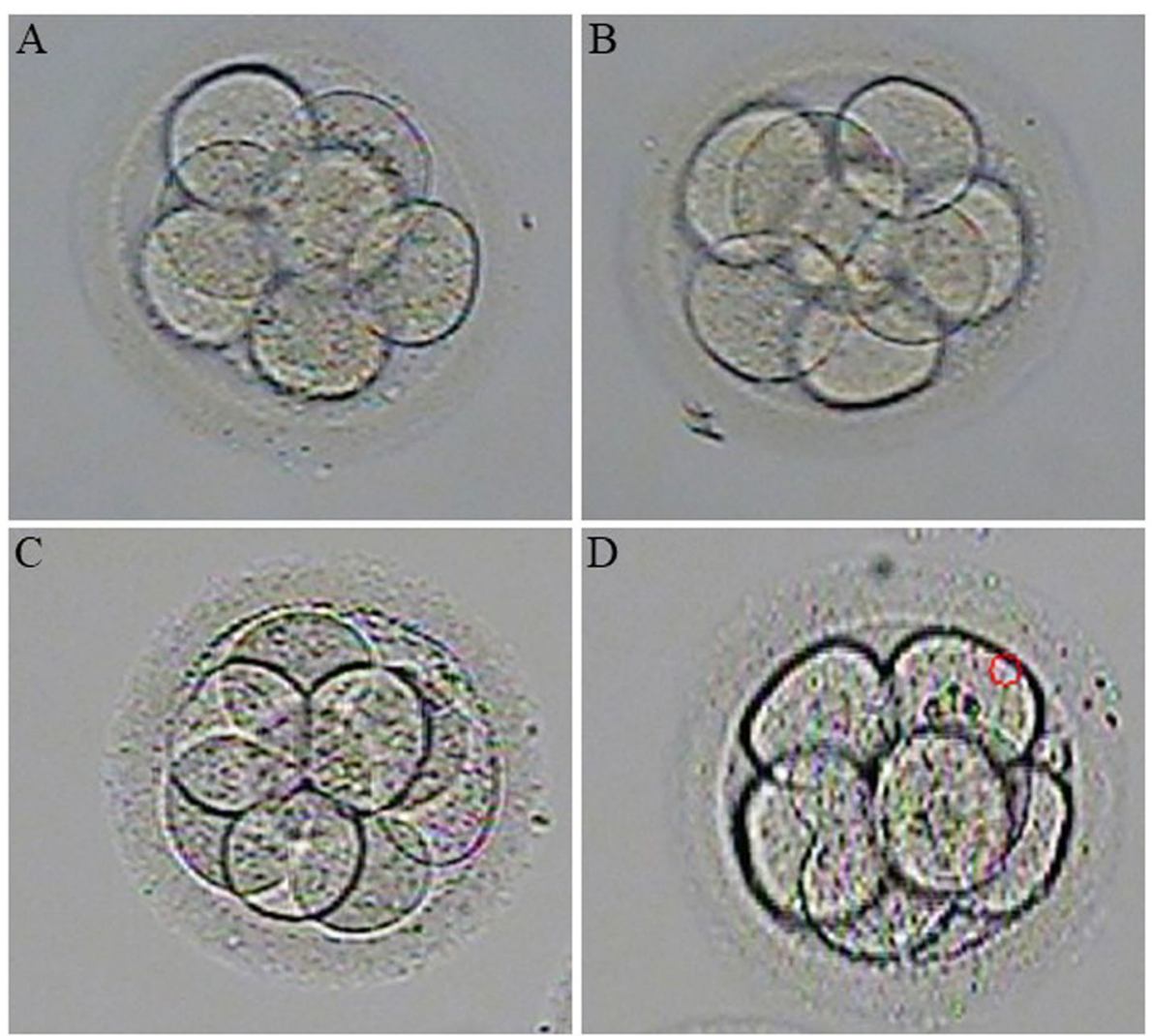

Fig. 1 Cleavage stage embryos before vitrification and after warming. A Embryo before vitrification with the Cryotop method. B Embryo before vitrification with the CryoTouch method. C Embryo after warming with the Cryotop method. D Embryo after warming with the CryoTouch method 
method (95\% CI 13.35-37.93), with no significant difference between the groups ( $p$ value $=0.5737)($ Table 3$)$.

\section{Discussion}

The present study detected no differences in post-warming survival rate, and clinical pregnancy, implantation and live birth rates of cleavage stage embryos that were undergone vitrification/warming procedures using two commercial methods, the CryoTouch method and Cryotop method.

Vitrification is the method of choice for embryo and gamete cryopreservation due to its simplicity, high efficiency, and low cost [12]. Although vitrification provides a high embryo survival rate and good clinical outcomes [6, 14], some concerns remain regarding the toxic effects of its ingredients which are used at high concentrations during manufacturing [21]. Most of the commercial vitrification media contain dimethylsulphoxide (DMSO), ethylene glycol (EG), propylene glycol (PG), or other polymers, all of which have some degrees of cellular toxicity [22]. The main goal during the formulation of vitrification media is to create a mixture of CPAs with the lowest toxicity [22]. The high concentration of CPAs needed for the best efficiency of vitrification accompanies with the increased probability of cryoprotectant toxicity [8]. Vitrification media of the Cryotop method contain DMSO and EG as CPAs [22, 23]. As declared by the company, vitrification media of the CryoTouch method also contain the same components. The observed similarity in embryo survival rate after vitrification/warming using the commercial media of these two companies somehow indicates their equality in efficiency for human embryo vitrification.

It has been revealed that prolonged exposure to CPAs enhances the probability of embryo toxicity during vitrification procedure $[24,25]$. Based on this, an optimal balance is required between the concentration of used CPAs and the speed of the procedure to obtain the best result. This delicate adjustment will minimize the likelihood of possible toxicity as well as osmotic injury which is prevalent when the embryos are held in media without mineral oil overlay during vitrification procedure [12]. The obvious difference between the vitrification protocols of CryoTouch and Cryotop methods is the incubation time of the equilibration solution as the CryoTouch method needs lesser incubation time compared to the Cryotop method (7 min for CryoTouch versus 10 minutes for Cryotop). This difference in incubation time may be due to different concentrations of cryoprotectants in two methods. However, this difference made no discrepancies in embryo survival rate and clinical outcomes.

Several vitrification protocols have been introduced so far; however, the reported clinical success rates are not homogeneous, possibly due to the technical differences
[14, 26, 27]. Cobo et al. (2012) [6] evaluated the qualities of 6019 embryos undergone vitrification/warming using the Cryotop method in different developmental stages and concluded that nearly $95 \%$ of the cleavage stage embryos had $100 \%$ of intact blastomeres. In line with this study, we showed $\sim 90 \%$ embryo survival rate using both CryoTouch and Cryotop methods. The Cryotop method for vitrification/warming is being widely used for human embryo and oocyte cryopreservation [13, 20, 28] and its efficiency is proven $[6,13,20,28]$. To the best of our knowledge, there is no published data from the CryoTouch method despite its routine use in infertility clinics of Iran, and data presented here show its comparable efficiency with the Cryotop method.

The number of reported live births obtained from vitrified/warmed human embryos has enormously increased over the past decades [13, 27]. However, different rates have been reported for implantation, clinical pregnancy, and live births. Cobo et al. reported an implantation rate of $34.6 \%$, a pregnancy rate of $41 \%$, and a live birth rate of $39.2 \%$ with vitrified/warmed embryos [6]. Desai et al. performed $200 \mathrm{FET}$ cycles with vitrified/warmed day 3 embryos in patients under 38 years old and reported $24 \%$ of implantation rate and $45 \%$ of clinical pregnancy rate [29]. Debrock et al. showed $16.1 \%$ of implantation rate and $20.7 \%$ of live birth rate in a randomized controlled trial conducted using vitrified/warmed embryos [29]. These high variable results in clinical outcomes may be explained by the differences in the possible contributors such as the developmental stage of transferred embryos, the selection criteria for vitrification, protocol of vitrification/warming, the selection criteria for ET, and patients' characteristics and medication protocols. La Marca et al. conducted a retrospective study which included 2617 embryos from 630 patients and showed $30.6 \%$ of live birth rate after the first FET on day 2 or 3 of embryonic development [13]. They used the Cryotop method for embryo vitrification/warming. Our results for clinical pregnancy, implantation, and live birth rates were all comparable with previous studies. In addition, we demonstrated no differences in implantation, clinical pregnancy, and live birth rates between embryos vitrified/warmed using CryoTouch and Cryotop commercial methods.

\section{Conclusions}

In conclusion, similar outcomes were observed in cleavage stage embryos vitrified/warmed using CryoTouch and Cryotop methods. These results provide strong evidence regarding the comparable efficiency of the CryoTouch versus Cryotop method. This is promising for infertility clinics in Iran that are using the CryoTouch method due to its availability, low cost, and prolonged expiration date as they are domestically produced in Iran. 


\begin{abstract}
Abbreviations
AMH: Anti-Mullerian hormone; ART: Assisted reproductive technology; ASRM: American Society for Reproductive Medicine; BMI: Body mass index; COCs: Cumulus cell-oocyte complexes; CPAs: Cryoprotective agents; DMSO: Dimethylsulphoxide; E2: Estradiol; EG: Ethylene glycol; ET: Embryo transfer; FET: Frozen embryo transfer; FSH: Follicle-stimulating hormone; GnRH: Gonadotropin-releasing hormone; hCG: Human chorionic gonadotropin; HRT: Hormone replacement therapy; ICSI: Intracytoplasmic sperm injection; IVF: In vitro fertilization; LH: Luteinizing hormone; OHSS: Ovarian hyperstimulation syndrome; OPU: Ovum pick-up; P: Progesterone; PG: Propylene glycol; RIF: Repeated implantation failure; SET: Single embryo transfer.
\end{abstract}

\section{Acknowledgements}

We acknowledge all staffs who worked in infertility clinics of Erfan Niayesh Hospital Tehran, Iran, and Mehr Fertility Research Center, Rasht, Iran.

\section{Authors' contributions}

SK, ADE, MM, RT: conceptualization; methodology; HV, MA, HH, SP: collected, analyzed, and interpreted the patients' data; SE, AG: drafted the manuscript; $H H$, SP: revised the manuscript critically for important intellectual content. All authors read and approved the final manuscript.

\section{Funding}

This work received no grant from any funding agency in the public, commercial, governmental, or academic sectors.

\section{Availability of data and materials}

The data that support the findings of this study are available from the infertility clinics.

\section{Declarations}

\section{Ethics approval and consent to participate}

This study was done according to the Ethical Principles for Medical Research and approved by the Institutional Review Board of Erfan Niayesh Hospital Tehran, Iran, and Mehr Fertility Research Center, Rasht, Iran. The committee's reference number is not available.

\section{Consent for publication}

Not applicable.

\section{Competing interests}

The authors declare that there are no competing interests related to the subject matter or materials discussed in this article.

\section{Author details \\ 'Infertility Clinic, Erfan Niayesh Hospital, Iran University of Medical Sciences, Tehran, Iran. ${ }^{2}$ Mehr Fertility Research Center, Guilan University of Medical Sciences, Rasht, Iran. ${ }^{3}$ Men's Health and Reproductive Health Research Center, Shahid Beheshti University of Medical Sciences, Tehran, Iran. ${ }^{4}$ Islamic Azad University, Rasht Branch, Rasht, Iran. ${ }^{5}$ College of Medicine, University of Illinois at Chicago, Chicago, IL, USA.}

Received: 23 February 2021 Accepted: 2 July 2021

Published online: 13 August 2021

\section{References}

1. Wennerholm U-B (2000) Cryopreservation of embryos and oocytes: obstetric outcome and health in children. Hum Reprod 15:18-25. https:// doi.org/10.1093/humrep/15.suppl_5.18

2. Veleva Z, Karinen P, Tomas C, Tapanainen JS, Martikainen H (2009) Elective single embryo transfer with cryopreservation improves the outcome and diminishes the costs of IVF/ICSI. Hum Reprod 24:1632-1639. https://doi. org/10.1093/humrep/dep042
3. Tiitinen A (2001) Elective single embryo transfer: the value of cryopreservation. Hum Reprod 16:1140-1144. https://doi.org/10.1093/humrep/ 16.6.1140

4. Saupstad M, Freiesleben NLC, Skouby SO, Andersen LF, Knudsen UB, Petersen KB, Husth M, Egeberg A, Petersen MR, Ziebe S, Andersen AN, Løssl K, Pinborg A (2019) Preparation of the endometrium and timing of blastocyst transfer in modified natural cycle frozen-thawed embryo transfers (mNC-FET): a study protocol for a randomised controlled multicentre trial. BMJ Open 9:e031811. https://doi.org/10.1136/bmjop en-2019-031811

5. Trounson A, Mohr L (1983) Human pregnancy following cryopreservation, thawing and transfer of an eight-cell embryo. Nature 305:707-709. https://doi.org/10.1038/305707a0

6. Cobo A, de los Santos MJ, Castellò D, Gámiz P, Campos P, Remohí J (2012) Outcomes of vitrified early cleavage-stage and blastocyst-stage embryos in a cryopreservation program: evaluation of 3,150 warming cycles, Fertil. Steril 98:1138-1146.e1. https://doi.org/10.1016/j.fertnstert.2012.07.1107

7. Serdarogullari M, Coban O, Boynukalin FK, Bilgin EM, Findikli N, Bahceci M (2019) Successful application of a single warming protocol for embryos cryopreserved by either slow freezing or vitrification techniques. Syst Biol Reprod Med 65:12-19. https://doi.org/10.1080/19396368.2018.1487477

8. Alcolak E, Abu Marar E, Mytas SC, Chalvatzas N, Palapelas V, Schöpper B, Diedrich K, Al-Hasani S (2011) Comparison of two different media for vitrification and rewarming of human zygotes: Prospective randomized study, Middle East Fertil. Soc J 16:189-193. https://doi.org/10.1016/j.mefs. 2011.02.003

9. European IVF-Monitoring Consortium (EIM), European Society of Human Reproduction and Embryology (ESHRE), Kupka MS, D'Hooghe T, Ferraretti AP, de Mouzon J, Erb K, Castilla JA, Calhaz-Jorge C, De Geyter C, Goossens V (2016) Assisted reproductive technology in Europe, 2011: results generated from European registers by ESHRE. Hum Reprod 31 (2):233-48. https://doi.org/10.1093/humrep/dev319

10. Wong KM, Mastenbroek S, Repping S (2014) Cryopreservation of human embryos and its contribution to in vitro fertilization success rates. Fertil Steril 102:19-26. https://doi.org/10.1016/j.fertnstert.2014.05.027

11. Desai N, Blackmon H, Szeptycki J, Goldfarb J (2007) Cryoloop vitrification of human day 3 cleavage-stage embryos: post-vitrification development, pregnancy outcomes and live births. Reprod Biomed Online 14:208-213. https://doi.org/10.1016/S1472-6483(10)60789-4

12. Fasano G, Fontenelle N, Vannin A-S, Biramane J, Devreker F, Englert Y, Delbaere A (2014) A randomized controlled trial comparing two vitrification methods versus slow-freezing for cryopreservation of human cleavage stage embryos. J Assist Reprod Genet 31:241-247. https://doi.org/10. 1007/s10815-013-0145-4

13. La Marca A, Dal Canto M, Buccheri M, Valerio M, MigniniRenzini M, Rodriguez A, Vassena R (2019) A novel transnational fresh oocyte donation (TOD) program based on transport of frozen sperm and embryos. Hum Reprod 34:285-290. https://doi.org/10.1093/humrep/dey331

14. Wirleitner B, Schuff M, Stecher A, Murtinger M, Vanderzwalmen P (2016) Pregnancy and birth outcomes following fresh or vitrified embryo transfer according to blastocyst morphology and expansion stage, and culturing strategy for delayed development. Hum Reprod 31:1685-1695. https://doi.org/10.1093/humrep/dew127

15. Zeng M, Su S, Li L (2018) Comparison of pregnancy outcomes after vitrification at the cleavage and blastocyst stage: a meta-analysis. J Assist Reprod Genet 35:127-134. https://doi.org/10.1007/s10815-017-1040-1

16. Cobo A, De Los Santos MJ, Castellò D, Gámiz P, Campos P, Remohí J (2012) Outcomes of vitrified early cleavage-stage and blastocyst-stage embryos in a cryopreservation program: Evaluation of 3,150 warming cycles, Fertil Steril. 98. https://doi.org/10.1016/j.fertnstert.2012.07.1107

17. Cobo A, Bellver J, Domingo J, Pérez S, Crespo J, Pellicer A, Remohí J (2008) New options in assisted reproduction technology: The Cryotop method of oocyte vitrification. Reprod Biomed Online 17:68-72. https://doi.org/ 10.1016/S1472-6483(10)60295-7

18. Kuwayama M (2007) Highly efficient vitrification for cryopreservation of human oocytes and embryos: The Cryotop method. Theriogenology 67:73-80. https://doi.org/10.1016/j.theriogenology.2006.09.014

19. Tsuribe PM, Coimbra IM, Vieira OL (2013) Implantation and pregnancy rates from vitrified embryos. JBRA Assist Reprod. 17. https://doi.org/10. 5935/1518-0557.20130078 
20. Braga DPAF, Setti AS, Figueira RCS, de C Azevedo M, laconelli A, Lo Turco EG, Borges E (2016) Freeze-all, oocyte vitrification, or fresh embryo transfer? Lessons from an egg-sharing donation program. Fertil Steril 106:615-622. https://doi.org/10.1016/j.fertnstert.2016.05.004

21. Kartberg A-J, Hambiliki F, Arvidsson T, Stavreus-Evers A, Svalander P (2008) Vitrification with DMSO protects embryo membrane integrity better than solutions without DMSO. Reprod Biomed Online 17:378-384. https://doi. org/10.1016/S1472-6483(10)60221-0

22. Mori C, Yabuuchi A, Ezoe K, Murata N, Takayama Y, Okimura T, Uchiyama K, Takakura K, Abe H, Wada K, Okuno T, Kobayashi T, Kato K (2015) Hydroxypropyl cellulose as an option for supplementation of cryoprotectant solutions for embryo vitrification in human assisted reproductive technologies. Reprod Biomed Online 30:613-621. https://doi.org/10.1016/j.rbmo. 2015.02.004

23. Mori C, Kuwayama M (2009) 167. The role of the cryoprotective effect of SSS on bovine oocytes during vitrification. Cryobiology 59:416-417. https://doi.org/10.1016/j.cryobiol.2009.10.181

24. AbdelHafez FF, Desai N, Abou-Setta AM, Falcone T, Goldfarb J (2010) Slow freezing, vitrification and ultra-rapid freezing of human embryos: a systematic review and meta-analysis. Reprod Biomed Online 20:209-222. https://doi.org/10.1016/j.rbmo.2009.11.013

25. Loutradi KE, Kolibianakis EM, Venetis CA, Papanikolaou EG, Pados G, Bontis I, Tarlatzis BC (2008) Cryopreservation of human embryos by vitrification or slow freezing: a systematic review and meta-analysis. Fertil Steril 90:186-193. https://doi.org/10.1016/j.fertnstert.2007.06.010
26. De Vos A, Van Landuyt L, Santos-Ribeiro S, Camus M, Van de Velde H, Tournaye H, Verheyen $\mathrm{G}$ (2016) Cumulative live birth rates after fresh and vitrified cleavage-stage versus blastocyst-stage embryo transfer in the first treatment cycle. Hum Reprod 31:2442-2449. https://doi.org/10.1093/ humrep/dew219

27. Debrock S, Peeraer K, Fernandez Gallardo E, De Neubourg D, Spiessens C, D'Hooghe TM (2015) Vitrification of cleavage stage day 3 embryos results in higher live birth rates than conventional slow freezing: a RCT. Hum Reprod 30:1820-1830. https://doi.org/10.1093/humrep/dev134

28. Liu H, Zhang J, Wang B, Kuang Y (2020) Effect of endometrial thickness on ectopic pregnancy in frozen embryo transfer cycles: an analysis including 17,244 pregnancy cycles. Fertil Steril 113:131-139. https://doi.org/10. 1016/j.fertnstert.2019.09.003

29. Desai N, AbdelHafez F, Bedaiwy MA, Goldberg J, Falcone T, Goldfarb J (2010) Clinical pregnancy and live births after transfer of embryos vitrified on day 3. Reprod Biomed Online 20:808-813. https://doi.org/10.1016/j. rbmo.2010.02.010

\section{Publisher's Note}

Springer Nature remains neutral with regard to jurisdictional claims in published maps and institutional affiliations.

\section{Submit your manuscript to a SpringerOpen ${ }^{\odot}$ journal and benefit from:}

- Convenient online submission

- Rigorous peer review

- Open access: articles freely available online

- High visibility within the field

- Retaining the copyright to your article

Submit your next manuscript at $\boldsymbol{\text { springeropen.com }}$ 